\title{
Ibero-American Expert Consensus on Squamous Cell Carcinoma of the Head and Neck Treatment in Patients Unable to Receive Cisplatin: Recommendations for Clinical Practice
}

\author{
Agustín Falco,' \\ Thiago Bueno de Oliveira, (ID) ${ }^{2}$ \\ Jon Cacicedo, ${ }^{3}$ Aylen Vanessa Ospina, ${ }^{4}$ \\ Miguel Ángel Ticona, ${ }^{5}$ Héctor Galindo, ${ }^{6}$ \\ Marcos David Pereira, José Luis Aguilar- \\ Ponce, ${ }^{8}$ Antonio Rueda-Domínguez, \\ Tannia Soria, ${ }^{10}$ Miren Taberna, ${ }^{1}$ \\ Lara Iglesias, ${ }^{12}$ Taysser Sowley, ${ }^{13}$ \\ Ricard Mesía (iD) 14 \\ On behalf of TTCC group (Spanish Group \\ for the Treatment of the Head and Neck \\ Cancer) \\ 'Instituto Alexander Fleming, Asociación \\ Argentina de Oncología Clínica (AAOC) \\ Buenos Aires, Argentina; ${ }^{2} \mathrm{AC}$ Camargo Cancer \\ Center, Sociedade Brasileira de Oncologia \\ Clínica (SBOC), São Paulo, Brazil; ${ }^{3}$ Hospital \\ Universitario Cruces/Biocruces Bizkaia Health \\ Research Institute, Barakaldo, Bizkaia, Spain; \\ ${ }^{4}$ ICCAL, Hospital Universitario Fundación \\ Santa Fe de Bogotá, Asociación Colombiana de \\ Hematología y Oncología (ACHO), Bogotá, \\ Colombia; ${ }^{5}$ Hospital Nacional Edgardo \\ Rebagliati Martins de Lima, Sociedad Peruana \\ de Oncología Médica (SPOM), Lima, Perú; \\ ${ }^{6}$ Pontificia Universidad Católica de Chile, \\ Sociedad Chilena de Oncología Médica \\ (SCOM), Santiago, Chile; ${ }^{7}$ Instituto de \\ Oncología Ángel H. Roffo, Asociación \\ Argentina de Oncología Clínica (AAOC), \\ Buenos Aires, Argentina; ${ }^{8}$ Instituto Nacional de \\ Cancerología, Sociedad Mexicana de Oncología \\ $(\mathrm{SMeO})$, Mexico City, Mexico; ' $U G C$ \\ Oncología Médica, Hospital Universitario \\ Regional y Virgen de la Victoria, IBIMA, Málaga, \\ Spain; ${ }^{10}$ Hospital SOLCA de Quito, Sociedad \\ Ecuatoriana de Oncología (SEO), Quito, \\ Ecuador; "Institut Català d'Oncologia, ICO \\ L'Hospitalet, Barcelona, Spain; ${ }^{12}$ Hospital 12 de \\ Octubre, Madrid, Spain; ${ }^{13}$ Instituto Oncológico \\ Nacional (ION) de Panamá, Sociedad Panameña \\ de Oncología (SPO), Panama City, Panama; \\ ${ }^{14}$ Institut Català d'Oncologia, ICO Badalona, \\ Barcelona, Spain
}

Correspondence: Ricard Mesía Institut Català d'Oncologia, ICO Badalona, Barcelona, Spain Email rmesia@iconcologia.net

\begin{abstract}
Cisplatin is the standard of treatment for squamous cell carcinoma of the head and neck (SCCHN) that has demonstrated efficacy, either in locally advanced disease when combined with radiotherapy at high doses, or in metastatic/recurrent disease when combined with other agents. However, the usual toxicities related to cisplatin, such as neurotoxicity, nephrotoxicity, ototoxicity, and hematologic toxicities, especially when high doses have been administered, have important implications in the patients' quality of life. The decision to administer cisplatin depends on several patient factors, such as age, performance status, weight loss, comorbidities, previous toxicities, chronic viral infection, or even the current SARS-CoV-2 pandemic. In order to establish recommendations for the management of patients with SCCHN, a group of experts in medical and radiation oncology from Spain and Latin-American discussed how to identify patients who are not candidates for cisplatin to offer them the most suitable therapeutic alternative.
\end{abstract}

Keywords: age, cisplatin, comorbidities, contraindication, frailty, toxicity

\section{Introduction}

Cisplatin is an essential component in the treatment of patients with squamous cell carcinoma of the head and neck (SCCHN), both in locally advanced and metastatic/recurrent disease. Phase III studies that enrolled a large patient population have demonstrated its efficacy, either in locally advanced disease when combined with radiotherapy at high doses $\left(100 \mathrm{mg} / \mathrm{m}^{2}\right.$ every three weeks $)$, or in metastatic disease when combined with other chemotherapy agents and biological or immuno-oncology therapies. ${ }^{1-5}$ However, cisplatin presents acute and late toxicity with important implications for patients' quality of life and may not be suitable in some patients. There are some patients in whom cisplatin is completely contraindicated, while others may only meet some of the criteria for not receiving it. In fact, only about half of the patients treated in clinical trials were able to complete the planned three cycles of high-dose cisplatin. ${ }^{6}$ To reduce the toxicity of radiotherapy with cisplatin, previous guidelines and consensus statements have recommended the use of highly conformal radiation techniques, such as intensitymodulated radiation therapy (IMRT) or volumetric modulated arc therapy (VMAT), or to use alternative treatments to high doses of cisplatin. ${ }^{7-9}$ 
In order to establish recommendations on the management of patients with SCCHN, a group of experts in medical and radiation oncology from Spain and Latin-American held several meetings to discuss the most relevant and recent scientific evidence. The objective of these discussions was to identify patients who are not candidates or who only meet some of the criteria for not receiving cisplatin in order to offer these patients the most suitable therapeutic alternative based on their clinical experience. This expert consensus is intended to guide and help health professionals when deciding on whether or not a patient is eligible to receive a drug, such as cisplatin, which has significant associated toxicities.

\section{Suitability for Cisplatin Chemotherapy}

The identification of predictive patient factors for acute and late toxicities may help to determine whether cisplatin should be administered or not, or whether it should be administered with careful monitoring. However, many of these factors alone do not always allow us to define an absolute contraindication to cisplatin. This section describes how to identify patients with SCCHN who may (are "fit" to) or may not (are "unfit" to) receive cisplatin, with special attention to those who meet only some of the criteria for not receiving cisplatin and where there are concerns about whether or not to administer it ("medium fit"). Table 1 shows a summary of the experts' recommendations for identifying these patients.

\section{Age}

The impact of age on the choice of treatment is highly influenced by other patient factors, such as life expectancy, comorbidities, performance status, social support, and patient preferences; therefore, therapeutic decisions should be based on biological rather than chronological age. ${ }^{10,11}$ For this reason, frailty has been established as a marker that is linked to biological age, and represents a crucial factor in making therapeutic decisions in elderly cancer patients. ${ }^{12}$ To assess the frailty of patients, several geriatric screening tools have been developed, such as the G8 and VES13 tools. ${ }^{13}$ These assessments allow for discrimination between "fit", vulnerable, and frail patients. Irrespective of chronological age, "fit" patients are able to tolerate high doses of cisplatin, while vulnerable patients require adaptive treatment, and frail patients are prevented from receiving curative therapy. ${ }^{12}$ Although both tools show reliable screening performance, the G8 is considered better for predicting "fit" patients, whereas the VES13 has a greater capacity to identify frail patients who are in need of comprehensive geriatric assessment. ${ }^{14}$ Nevertheless, as the G8 has identified twice as many vulnerable patients as the Karnofsky index, it is the method of choice in patients aged $\geq 70$ years. ${ }^{11}$ Although these screening tools cannot replace comprehensive geriatric assessment, they may be useful to identify patients who are eligible for comprehensive geriatric assessment.

Several consensus statements have established a cut-off point of 70 years as the age at which cisplatin administration is not recommended, under the rationale that no benefit is obtained by adding chemotherapy to radiotherapy after that age. ${ }^{7,15}$ However, this limitation has been changed, as several reports have shown that patients aged $\geq 70$ years can achieve the same clinical benefit from cisplatin as younger patients. ${ }^{12,16-19}$ Therefore, none of these consensus papers have stated that age by itself is a criterion for which cisplatin therapy is absolutely contraindicated. $^{7,12}$

What is known is that addition of cisplatin to radiotherapy increases the toxicity of treatment and the consumption of healthcare resources, including emergency department visits and inpatient admissions..$^{2,20,21}$ The incidence of this toxicity is significantly higher in people $>70$ years of age, with higher rates of thrombocytopenia, diarrhea, and grade $\geq 3$ stomatitis. ${ }^{17}$ In contrast, a retrospective study showed that the rate of acute toxicities was not higher in older versus younger patients $\left(<65\right.$ years). ${ }^{22}$

\section{Performance Status}

According to a consensus statement that established recommendations for the use of platinum-based therapy in patients with SCCHN across several categories, having an Eastern Cooperative Oncology Group (ECOG) performance status of 2 was considered a high risk factor for developing platinum intolerance, while an ECOG $\geq 3$ was an absolute contraindication for platinum-based therapy, meaning these patients are only candidates for palliative treatment (Table 2). The rationale for this recommendation was that patients with reduced performance status (ECOG $\geq 2$ ) have low therapeutic adherence due to fear of an increased risk of chemotherapy-related toxicity. ${ }^{7}$

Based on a meta-analysis that analyzed clinical trials comparing locoregional treatment with locoregional treatment plus chemotherapy, evidence for the effect of induction chemotherapy according to performance status on patient survival remains unclear. ${ }^{15}$ Regarding the appearance of late severe toxicity after concomitant 
Table I Summary of Expert Recommendations for Identifying SCCHN Patients with Absolute or Relative Contraindications to Cisplatin Administration or Who Do Not Meet All of the Criteria

\section{Age}

- Biological age must prevail over chronological age in order to establish therapeutic decisions.

- Chronological age alone should not be an absolute contraindication for receiving platinum; and it should not limit the administration of cisplatin to full doses.

- Patients over 70 years of age are at greater risk of toxicity and non-cancer-related mortality, and there may be no benefit from cisplatin treatment.

However, frailty seems to be a stronger risk than age alone, and should always be considered when making treatment decisions.

- Geriatric screening tools (G8 or VESI3) allow for discrimination between "fit", vulnerable, and frail patients.

- G8 geriatric screening tool is better for predicting "fit" patients, whereas VESI3 has a higher capacity for identifying frail patients in need of comprehensive geriatric assessment.

- G8 geriatric screening tool should be the method of choice for patients under 70 years of age, as it has been able to identify twice as many vulnerable patients as the Karnofsky index.

- Regardless of chronological age, "fit" patients may be able to tolerate high doses of cisplatin and get similar benefit as the general population, and they should be treated with curative intent.

\section{Performance status}

- Despite scarce evidence, ECOG $>2$ is an absolute contraindication to platinum administration, while ECOG = 2 is a relative contraindication.

- Assessment of each individual patient will determine whether performance status is influenced by the disease itself, by the treatment, or by the comorbidities. If performance status is influenced by:

- The disease, then treatment should be administered;

- The on-going treatment, then treatment should be adjusted;

- Comorbidities, then treatment should not be administered.

\section{Weight loss}

- Weight loss of $>30 \%$ is an absolute contraindication to cisplatin treatment.

- Weight loss of $20-30 \%$ is a relative contraindication, and other factors should be considered.

- Nutritional assessment should be made before making a therapeutic decision using validated screening and assessment tools, such as the Subjective Global Assessment tool, the patient-generated Subjective Global Assessment tool, and the Malnutrition Screening tool.

\section{Comorbidities}

- It is important to define previous comorbidities (and their clinical impact) when making treatment decisions in the curative or palliative setting.

- Functional tests with well-established cut-off points should be performed in all patients, including audiometry, renal function, echocardiogram, lung function,

and nutritional status.

- These tests would be advisable for both asymptomatic patients and those with previous history of these comorbidities.

- Depending upon the severity of the comorbidity, an absolute contraindication may be established (see Table 2).

- For relative contraindications due to comorbidities, it is necessary to take into account the additive effect of several lower severity comorbidities, which can mean an absolute contraindication of treatment.

- Patients with > I relative contraindication should be analyzed individually by the multidisciplinary team in order to establish the best treatment.

- In situations where toxicity may be a problem for the patient, despite initially meeting the requirements for cisplatin, closer monitoring should be established.

\section{Chronic viral infections}

- HIV and hepatitis B and C virus screening is recommended for all patients who are scheduled to receive cisplatin. Additionally, depending on the endemic areas, it is also advisable to screen for HTLV-I.

- In patients with an active hepatitis B or C infection, liver function status should be checked to see if antiviral administration is appropriate. If so, chemotherapy should be started.

- It is necessary to perform cost-effectiveness studies to evaluate the need for screening of these viruses in the oncological population.

\section{COVID-19}

- Screening for SARS-CoV-2 virus is advisable in all patients who will undergo surgery, systemic therapy, or radiotherapy.

- In patients with locally advanced SCCHN, radiotherapy with curative intent should only be interrupted in patients with severe COVID-19, but chemotherapy should be interrupted in all patients, regardless of whether they are asymptomatic or have mild or severe symptoms.

- In patients with recurrent and metastatic SCCHN, treatment should be interrupted in all patients with COVID-19, regardless of whether they are asymptomatic or have mild or severe symptoms.

- In the palliative treatment scenario, it is necessary to consider age, comorbidities, and the risk-benefit ratio when deciding whether to continue treatment. In addition, this decision must be discussed with the patient. 
Table I (Continued).

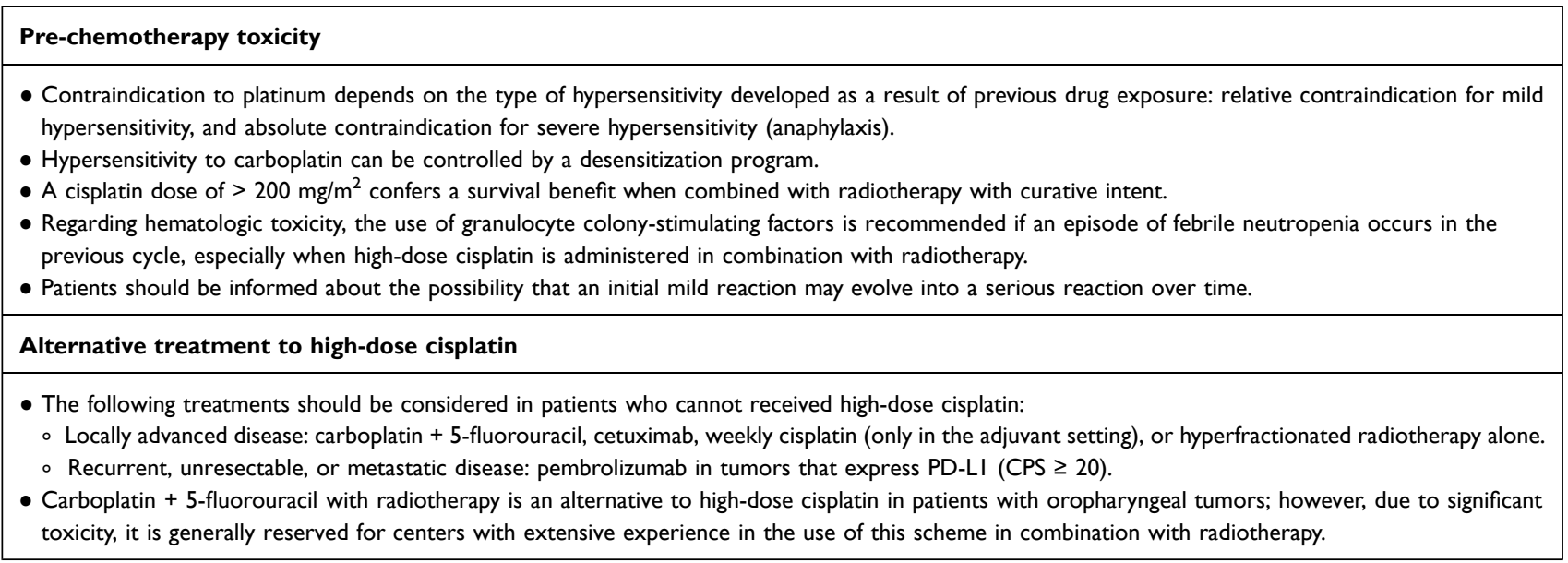

Abbreviations: CPS, combined positivity score; HIV, human deficiency virus; HTLV, human T-cell leukemia-lymphoma virus; PD-LI, programmed-death ligandI.

chemotherapy plus radiotherapy in patients with locally advanced SCCHN, another study found that there was no difference according to Karnofsky index performance status. ${ }^{23}$ For this reason, when using the Karnofsky performance status score, clinicians should ensure that patients with disabilities have equal access to treatment, as they may have difficulties with activities of daily living that are unrelated to their SCCHN prognosis. In such cases, an appropriate assessment of performance status should be made, taking into account the individual's usual functional ability and their need for assistance with activities of daily living. $^{7}$

\section{Weight Loss}

An involuntary weight loss of $\geq 20 \%$ has been established as a relative contraindication for receiving platinum-based treatment (Table 2). ${ }^{7}$ Nutritional deficiency and weight loss are important risk factors for receiving cisplatin. Weight loss has been shown to influence clinical outcomes in patients with SCCHN who receive chemoradiotherapy, with a weight loss of $\geq 20 \%$ before diagnosis being significantly correlated with treatment interruption, infections, early mortality, increased hospital readmission, and reduced survival. ${ }^{24}$ Therefore, an early nutritional approach to patients with SCCHN can reduce weight loss and improve outcomes. ${ }^{25}$

Weight loss in the 6 months prior to treatment does not appear to affect the development of late chemoradiotherapy-related toxicity. ${ }^{23}$ However, pretreatment weight loss is associated with treatment failure, locoregional recurrence, distant metastases, and reduced overall survival
(OS). The greater the weight loss, the worse the outcome. However, none of these outcomes seem to be affected if weight loss occurs during treatment. ${ }^{26}$

Despite intense nutritional support following diagnosis and throughout treatment, patient nutritional status deteriorates during radiotherapy. However, induction chemotherapy may help improve nutritional status by reducing symptoms that limit oral intake. ${ }^{27}$ Thus, a nutritional assessment before, during, and after treatment is essential to minimize symptoms or adverse effects that may arise. Some of the nutritional screening and assessment tools available and validated in cancer patients are the Subjective Global Assessment Tool, the patientgenerated Subjective Global Assessment, and the Malnutrition Screening Tool. ${ }^{28}$

\section{Comorbidities}

Given the usual cisplatin-related toxicities, such as neurotoxicity, nephrotoxicity, ototoxicity, and hematologic toxicities, especially when high doses have been administered,,$^{9,29,30}$ the suitability of cisplatin administration will depend on the presence of comorbidities that may be exacerbated. Because performance status is not a reliable substitute for assessing comorbidities as a prognostic measure, different tools have been developed to measure and quantify patient comorbidities. Some of the more commonly used assessment tools are the Adult Comorbidity Evaluation and the Charlson Comorbidity Index. $^{31,32}$

According to previous consensus statements, some of the main comorbidities that are absolute contraindications 
Table 2 Absolute and Relative Contraindications to Cisplatin Administration in Patients with SCCHN

\begin{tabular}{|c|c|}
\hline Absolute Contraindications & Relative Contraindications \\
\hline $\begin{array}{l}\text { - ECOG of } 3-4 \text {. } \\
\text { - Weight loss of }>30 \% \text {. } \\
\text { - Renal failure (creatinine clearance }<40 \mathrm{~mL} / \mathrm{min} \text { ). } \\
\text { - Hearing disorders: previous hearing loss or grade } \geq 2 \text { tinnitus. } \\
\text { - Neurologic disorders: grade } \geq 2 \text { diabetic neuropathy, peripheral sensory } \\
\text { neuropathy, orthostatic hypotension, Lhermitte's sign, seizures, focal } \\
\text { encephalopathy. } \\
\text { - Pregnancy or lactation. } \\
\text { - Child-Pugh B or C liver disease. } \\
\text { - Platelet count }<1 \times 10^{5} / \mathrm{mm}^{3} \text {. } \\
\text { - Neutrophil count }<1500 / \mathrm{mm}^{3} \text {. } \\
\text { - Hemoglobin level }<9 \mathrm{~g} / \mathrm{dl} \text {. } \\
\text { - Congestive heart failure } \mathrm{NYHA} \text { Class III or IV despite cardiovascular } \\
\text { optimization therapy. } \\
\text { - Congestive heart failure NYHA Class I or II with LVEF } \leq 50 \% \text {. } \\
\text { - Hepatitis B or C virus infection without antiviral treatment. } \\
\text { - HIV infection with CD4 cell count }<200 / \mu \mathrm{L} \text {, detectable viral load, and } \\
\text { acquired immunodeficiency syndrome. } \\
\text { - Hypersensitivity to platinum-based therapy. } \\
\text { - Asymptomatic or symptomatic COVID-I9 confirmed. }\end{array}$ & $\begin{array}{l}\text { - ECOG of } 2 \text {. } \\
\text { - Involuntary weight loss of } 20-30 \% \text {. } \\
\text { - Creatinine clearance of } 40-60 \mathrm{~mL} / \mathrm{min} \text {. } \\
\text { - Grade I hearing or neurologic disorders. } \\
\text { - Grade } \geq 2 \text { bone marrow, liver, and lung disorders. } \\
\text { - Hypertension, previous heart disease, diabetes, and recurrent } \\
\text { pulmonary infections. } \\
\text { - Concomitant use of nephrotoxic drugs. } \\
\text { - HIV infection with CD4 cell count } 200-350 / \mu \mathrm{L} \text {. } \\
\text { - Previous platinum treatment }\left(>200 \mathrm{mg} / \mathrm{m}^{2} \text { or }>3 \text { induction }\right. \\
\text { cycles with docetaxel, carboplatin, and } 5 \text {-fluorouracil). } \\
\text { - Cumulative cisplatin dose of }>200 \mathrm{mg} / \mathrm{m}^{2} \text { or having received }>3 \\
\text { induction cycles with docetaxel, cisplatin, and } 5 \text {-fluorouracil. }\end{array}$ \\
\hline
\end{tabular}

Abbreviations: ECOG, Eastern Cooperative Oncology Group; HIV, human deficiency virus; LVEF, left ventricular ejection fraction; NYHA, New York Heart Association.

to cisplatin administration are renal failure (creatinine clearance $<50 \mathrm{~mL} / \mathrm{min}$ ), hearing disorders (previous hearing loss or tinnitus grade $\geq 2$ ), neurologic disorders (grade $\geq 2$ diabetic neuropathy, peripheral sensory neuropathy, orthostatic hypotension, Lhermitte's sign, seizures, or focal encephalopathy), hypersensitivity to platinum-based therapy, and pregnancy or lactation (Table 2). ${ }^{7,8}$ Other absolute contraindication criteria are Child-Pugh B or C, platelets $<1 \times 10^{5} / \mathrm{mm}^{3}$, neutrophils $<1500 / \mathrm{mm}^{3}$, hemoglobin $<9 \mathrm{~g} / \mathrm{dl}$, hepatitis $\mathrm{B}$ or $\mathrm{C}$ without antiviral treatment, congestive heart failure New York Heart Association (NYHA) class III or IV despite cardiovascular optimization therapy, and congestive heart failure NYHA class I or II with left ventricular ejection fraction $(\mathrm{LVEF}) \leq 50 \%$. In addition, although high blood pressure is not a contraindication to cisplatin administration by itself, it may carry an increased risk of developing acute or chronic renal failure. Patients with more advanced cardiomyopathies, including heart failure, are more susceptible to developing cardiorenal syndrome. ${ }^{34}$

Many of these absolute contraindication criteria can become relative contraindications, or high risk, depending on how they develop. Thus, relative contraindication criteria are: receiving cisplatin with a creatinine clearance of 50-60 $\mathrm{mL} / \mathrm{min}$; grade 1 hearing or neurologic disorders; grade $\geq 2$ bone marrow, liver and lung disorders; hypertension, previous heart disease, diabetes, and recurrent pulmonary infections; previous platinum treatment $(>$ $200 \mathrm{mg} / \mathrm{m}^{2}$ or more than three induction cycles with docetaxel, carboplatin, and 5-fluorouracil); or concomitant use of nephrotoxic drugs (Table 2). ${ }^{7,8}$

\section{Chronic Viral Infections}

Chronic viral infections that can condition the administration of platinum-based therapy are those caused by human immunodeficiency virus (HIV) and hepatitis B or $\mathrm{C}$ virus. According to previous consensus statements, cisplatin is completely contraindicated in patients with HIV, with CD4 cells $<200 / \mu \mathrm{L}$, with detectable viral load, and with acquired immunodeficiency syndrome. However, if the CD4 cell count is between 200 and $350 / \mu \mathrm{L}$, the contraindication to cisplatin is only relative (Table 2). ${ }^{7-9,35}$ The main rationale for these recommendations is the weakening of the immune system by HIV. Another factor to consider is that some antiretroviral drugs used by patients with HIV are inducers or inhibitors of certain cytochrome enzymes, which can influence the effectiveness or tolerability of cisplatin or other concomitant treatments, such as antiemetics and corticosteroids. $^{36}$ 
In chronic active hepatitis $\mathrm{B}$ or $\mathrm{C}$ virus infection, contraindication to cisplatin administration is absolute in patients who are not receiving antiviral treatment (Table 2). ${ }^{8,35}$ This is due to the possibility of exacerbation of hepatitis during cancer treatment. Many chemotherapeutic agents, including cisplatin and carboplatin, are associated with reactivation of hepatitis $\mathrm{B}$ virus. ${ }^{37}$ To avoid virus reactivation in patients scheduled to receive chemotherapy, screening for virus surface antigen (HBsAg), anti-surface antigen antibodies (antiHBs), and anti-core antibodies (anti-HBc) is recommended for all patients. ${ }^{37}$ In patients with an active infection (HBsAg-positive) liver function status would be checked. If liver function allows for antiviral administration, chemotherapy would be started. In patients with resolved hepatitis $\mathrm{B}$ infection (HBsAg-negative and anti-HBc-positive), liver function and virus DNA should be measured before starting antiviral prophylaxis, after which chemotherapy can be initiated. ${ }^{37}$

Because curative treatment for hepatitis $\mathrm{C}$ is now available, the number of patients with this infection is expected to decrease. Even so, several retrospective studies have detected a higher rate of hepatitis $\mathrm{C}$ infection in patients with SCCHN than in the general population, although with a similar prognosis. ${ }^{38,39}$ Because of this, it is important to screen for this virus in patients with SCCHN before chemotherapy administration.

\section{COVID-19}

The SARS-CoV-2 virus pandemic, which causes COVID19, has disrupted all health programs and protocols at all levels, including in patients with SCCHN, by interrupting, delaying, or discontinuing treatment. As the pandemic evolves, and as more information about the virus becomes available, it will be necessary to determine whether measures taken to address these disruptions are temporary or permanent.

Depending on the severity of COVID-19, chemotherapy administration may increase mortality risk in cancer patients. However, administration of chemotherapy or other anticancer treatments does not increase the risk of death from COVID-19, but rather that the mortality risk depends primarily on the patient's age, sex, and comorbidities. ${ }^{40}$ Although patients receiving chemotherapy have a higher risk of death from COVID-19 than from immunotherapy, radiotherapy, or targeted therapy, the difference in mortality risk was not statistically significant. The risk of death in patients receiving palliative chemotherapy is higher than in those receiving nonpalliative chemotherapy ( $35 \%$ vs $16 \%) .^{40}$

Various recommendations have been established for patients with SCCHN and COVID-19. ${ }^{41-43}$ These recommendations all agree on the importance of individualizing therapy and not delaying or interrupting treatment in patients without virus infection. They also agree that systemic therapy should be avoided in patients aged $>70$ years or in younger patients with high-risk comorbidities (ie diabetes or cardiovascular disease); induction therapy with cisplatin should avoided, since this strategy has not been shown to provide added benefit over that provided by standard radiochemotherapy; and total treatment time should be reduced. ${ }^{41-43}$ In patients who are not candidates for platinum-based therapy, these guidelines recommend that exclusive radiotherapy be prioritized and that suitability of concomitant cetuximab administration be assessed on an individual patient basis. ${ }^{43}$

\section{Pre-Chemotherapy Toxicity}

The risk of cisplatin toxicity increases due to the accumulation of cisplatin doses over time, which can remain in the plasma for up to 20 years. Therefore, previous cisplatin administration must be taken into account in order to avoid excessive drug accumulation and serious toxicities. ${ }^{44}$

Neurotoxicity can occur with cumulative cisplatin doses of $\geq 300 \mathrm{mg} / \mathrm{m}^{2}$, especially in patients with a history of drug and alcohol use, diabetes, older age, and elevated serum creatinine levels. ${ }^{9}$ The incidence of clinical neurotoxicity with cisplatin does not seem to be directly related to the response rate. ${ }^{30}$ This detail is important because, although cisplatin use is associated with the risk of neurotoxicity, this does not have to necessarily be a problem as their symptoms could be prevented or mitigated. ${ }^{30}$

Ototoxicity may occur with cumulative cisplatin doses of $60 \mathrm{mg} / \mathrm{m}^{2}$, especially in patients with previous hearing impairment, older age, some genetic polymorphisms (megalin, ACYP2, TPMT, COMT, XPC) or systemic inflammation. ${ }^{9}$ It is known that from $300 \mathrm{mg} / \mathrm{m}^{2}$ of cisplatin, the risk of ototoxicity increases by $5 \%$ for every $100 \mathrm{mg} / \mathrm{m}^{2}$ accumulated. ${ }^{29}$

Nephrotoxicity can arise from cumulative cisplatin doses of $50 \mathrm{mg} / \mathrm{m}^{2}$; risk factors for nephrotoxicity include low ECOG performance status, regular use of nonsteroidal anti-inflammatory drugs, older age, tobacco use, hypoalbuminemia, and a previous glomerular filtration rate $<$ $60 \mathrm{~mL} / \mathrm{min} / 1.73 \mathrm{~m}^{2} .9$ 
Table 3 Cumulative Doses and Risk Factors Associated with Cisplatin Toxicity

\begin{tabular}{|c|c|c|c|}
\hline Cumulative Dose of Cisplatin & Toxicity & Risk Factors & Test \\
\hline$\geq 300 \mathrm{mg} / \mathrm{m}^{2}$ & Neurotoxicity & $\begin{array}{l}\text { - Drug } \\
\text { - Alcohol } \\
\text { - Diabetes } \\
\text { - Older age } \\
\text { - High serum creatinine }\end{array}$ & $\begin{array}{l}\text { - Vibration perception test } \\
\text { - Deep tendon reflexes }\end{array}$ \\
\hline $60 \mathrm{mg} / \mathrm{m}^{2}$ & 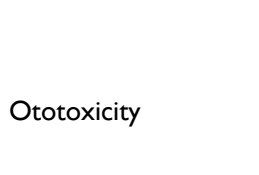 & $\begin{array}{l}\text { - Hearing impairment } \\
\text { - Older age } \\
\text { - Genetic polymorphism } \\
\text { - Systemic inflammation }\end{array}$ & - Audiometric tests \\
\hline $50 \mathrm{mg} / \mathrm{m}^{2}$ & 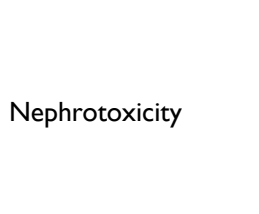 & $\begin{array}{l}\text { - Low ECOG PS } \\
\text { - Regular NSAIDs } \\
\text { - Tobacco } \\
\text { - Hypoalbuminemia } \\
\text { - GFR }<60 \mathrm{~mL} / \mathrm{min} / 1.73 \mathrm{~m}^{2}\end{array}$ & $\begin{array}{l}\text { - Creatinine clearance } \\
\text { - Serum creatinine level }\end{array}$ \\
\hline Any dose & Hematologic toxicity & $\begin{array}{l}\text { - Older patients } \\
\text { - Pre-existing anemia }\end{array}$ & - Blood tests \\
\hline
\end{tabular}

Abbreviations: ECOG, Eastern Cooperative Oncology Group; GFR, glomerular filtration rate; NSAID, non-steroidal anti-inflammatory drugs; PS, performance status.

Finally, hematologic toxicity, which can arise from any dose, is common in older patients and those with preexisting anemia. ${ }^{9}$ The development of any of these adverse events will determine the next treatment administered.

To mitigate or reduce the risk of these toxicities, in addition to taking into account previous cisplatin administrations, laboratory tests to detect for the presence or worsening of toxicities should be conducted. For example, to test for neurotoxicity, vibration perception tests or exploration of deep tendon reflexes can be done. Audiometric tests should be performed for ototoxicity. For nephrotoxicity, the rate of creatinine clearance or serum creatinine levels should be measured, and a complete blood count for hematologic toxicity (Table 3). ${ }^{9}$

According to previous consensus statements, an cumulative cisplatin dose of $>200 \mathrm{mg} / \mathrm{m}^{2}$ or more than three induction cycles with docetaxel, cisplatin, and 5-fluorouracil has been established as a relative contraindication for further cisplatin administration (Table 2) ${ }^{7-9,35,44}$ However, it should be noted that a cisplatin dose of $>200 \mathrm{mg} / \mathrm{m}^{2}$ confers a survival benefit when combined with radiotherapy with curative intent. ${ }^{45}$

An absolute contraindication to cisplatin is hypersensitivity to platinum-based therapy (Table 2)..$^{7,85,46}$ The incidence and severity of hypersensitivity to platinum varies from one agent to another. While cisplatin or carboplatin can cause type I (early-onset, IgE-mediated) or type IV (late-onset, T-cell-mediated) hypersensitivity, oxaliplatin primarily leads to type I hypersensitivity, but also type II and III hypersensitivity. ${ }^{46}$

\section{Alternative Treatments to High-Dose Cisplatin Locally Advanced Disease}

In patients with locally advanced SCCHN who are not candidates for high-dose cisplatin, current National Comprehensive Cancer Network (NCCN) guidelines recommend primary systemic treatment plus concurrent radiotherapy with carboplatin +5 -fluorouracil (category 1 , preferred regimen), cetuximab (category $2 \mathrm{~B}$, useful in certain circumstances) or cisplatin $40 \mathrm{mg} / \mathrm{m}^{2}$ weekly (category $2 \mathrm{~B}) .{ }^{47}$ According to the Spanish Society of Medical Oncology (SEOM), radiotherapy alone should be considered in patients with locally advanced disease who are not candidate for platinum-based chemotherapy (Quality of evidence I, strength of recommendation A). ${ }^{48}$

The combination of carboplatin and 5-fluorouracil administration with radiotherapy was supported by a phase III study that demonstrated its superiority in both OS and locoregional control compared with radiotherapy alone in patients with advanced oropharyngeal carcinoma. ${ }^{49}$ Although there are no direct comparisons, a subsequent retrospective analysis showed median OS 
was prolonged in patients treated with cisplatin compared with those receiving carboplatin +5 -fluorouracil. In addition, adherence was lower with carboplatin +5 -fluorouracil, possibly because of its higher rates of toxicity. ${ }^{50}$ A meta-analysis also showed that OS was shorter with carboplatin than with cisplatin, with more hematologic toxicity and mucositis. ${ }^{51}$

Clinical evidence supporting the cetuximab use as an alternative to cisplatin in locally advanced disease was provided by a phase III study, in which the use of radiotherapy with or without cetuximab was evaluated. The combination of cetuximab and radiotherapy significantly prolonged locoregional control versus radiotherapy alone (24.4 vs 14.9 months, $p=0.005)$ and significantly increased median OS (49.0 vs 29.3 months, $p=0.03$ ) without increasing toxicity associated with radiotherapy. ${ }^{52,53}$ However, in a later study, the combination of radiotherapy and cetuximab led to lower rates of 5-year progression-free survival (PFS; 67\% vs 78\%) and OS (78\% vs $85 \%)$ compared with radiotherapy and highdose cisplatin. ${ }^{3}$ A previous study showed that only $27.5 \%$ of patients received three-weekly cisplatin and radiotherapy for locally advanced SCCHN treatment. ${ }^{54}$ The combination of cetuximab and paclitaxel may be a treatment option in patients with recurrent/metastatic disease for whom platinum is contraindicated, showing an overall response rate of 54\% ( $22 \%$ complete response), a disease control rate of $80 \%$, a median PFS of 4.2 months, and a median OS of 8.1 months. ${ }^{55}$ However, this combination is not yet approved by the European Medicines Agency (EMA) or the Food and Drug Administration (FDA) for this indication.

The use of weekly cisplatin has also been tested as an alternative to high-dose cisplatin. The combination of weekly cisplatin $50 \mathrm{mg}$ and postoperative radiation therapy improved locoregional control and survival of patients with locally advanced SCCHN versus radiation therapy alone without significantly increasing the rate of late radiation complications in this group. ${ }^{56}$ However, cisplatin $100 \mathrm{mg} / \mathrm{m}^{2}$ administered once every 3 weeks resulted in superior locoregional control in the adjuvant setting, albeit with higher rates of toxicity, than did cisplatin $30 \mathrm{mg} / \mathrm{m}^{2}$ administered once a week. ${ }^{57}$ On the other hand, recent publications showed that weekly cisplatin $40 \mathrm{mg}$ / $\mathrm{m}^{2}$ plus radiotherapy is non-inferior to cisplatin $100 \mathrm{mg} / \mathrm{m}^{2}$ once every 3 weeks plus radiotherapy in patients with postoperative high-risk locally advanced SCCHN and has a favorable toxicity profile. ${ }^{58,59}$ Thus, weekly cisplatin plus radiotherapy could be considered as an option for these patients in the adjuvant setting.

Another alternative to high-dose cisplatin is hyperfractionated radiotherapy. ${ }^{60}$ Hyperfractionation is considered as standard-of-care in patients with locally advanced SCCHN undergoing definitive radiotherapy, as it has demonstrated an improvement in OS compared with conventional fractionated radiotherapy. ${ }^{60}$

\section{Recurrent, Unresectable, or Metastatic Disease}

In patients with recurrent, unresectable, or metastatic disease with no surgery or radiotherapy option, the preferred alternative regimen to cisplatin according to NCCN is pembrolizumab as first-line therapy (for tumors that express PD-L1 with a combined positivity score [CPS] $\geq$ 1; category 1 if CPS $\geq 20$ ), ${ }^{47}$ and according to SEOM the combination of paclitaxel plus cetuximab (ERBITAX) should be considered (IIB). ${ }^{48}$

The efficacy of pembrolizumab was demonstrated in the KEYNOTE-048 trial, an open-label, phase III study in which patients were randomized to pembrolizumab alone, pembrolizumab plus a platinum and 5-fluorouracil, or cetuximab plus a platinum and 5-fluorouracil. ${ }^{5}$ Pembrolizumab monotherapy improved OS versus cetuximab plus platinum and 5-fluorouracil in the combined positivity score (CPS) $\geq 20$ (median 14.9 vs 10.7 months, $p=0.0007)$ and CPS $\geq 1$ (12.3 vs 10.3 months, $p=$ $0.0086)$ patient populations, and was non-inferior in the total population (11.6 vs 10.7 months). ${ }^{5}$

\section{Supportive Measures to Improve the Treatment of Patients with SCCHN}

In addition to the therapeutic alternatives described in the previous section, all patients with $\mathrm{SCCHN}$ also require a series of supportive measures (described below). Table 4 provides a summary of expert recommendations about supportive measures in these patients.

\section{Socioeconomic Factors: Family Support, Cultural Factors, Educational Level}

Socioeconomic factors and family support are critical factors in ensuring safety and adequate adherence to both high-dose cisplatin treatment and radiotherapy. ${ }^{61}$ Social considerations include the patient's understanding and willingness to undergo the recommended treatment, family and social support during and after treatment, and health 
Table 4 Summary of Expert Recommendations for Supportive Measures to Improve the Treatment of Patients with SCCHN

Socioeconomic Factors

- In order to define treatment administration for organ preservation in SCCHN, the characteristics of the healthcare institutions, health system, clinical conditions of the patient and other socioeconomic factors must be taken into account to make the best treatment choice for each case.

- Socioeconomic factors are associated with advanced clinical status at initial diagnosis of SCCHN; in addition, presence of comorbidities are important predictors of quality of life in survivors. All of these should be considered when deciding the best therapeutic approach for each patient.

- Given the nature of the disease, patients will likely require a regular caregiver; therefore, it is advisable to develop education programs specifically for patients' caregivers.

- After treatment, it is important to take an individualized rehabilitation approach to guide and help patients to return to work and resume their daily lives.

- Clinical and social variables of each patient should be used to better define candidates for intensive rehabilitation and comprehensive social support, with the goal of reducing work disability.

\section{Multidisciplinary Approach}

- A multidisciplinary approach is essential for the management of patients with SCCHN. Patients should be managed by a multidisciplinary team at all times until survivorship is reached.

- Specialized nurses ensure the coordination of multimodal treatment and its teams, the continuous assistance of patients and their caregivers, and the early detection of toxicity or potentially serious conditions. Advanced nurses should be recommended as part of the multidisciplinary team for SCCHN management.

- Nutritional status should be assessed by a specialist before, during, and at the end of treatment. Adequate nutritional function can favorably impact treatment outcomes, as well as recovery from toxicity.

- Nutritional assessment is advisable in patients who, at diagnosis, do not appear to be malnourished or have weight loss, as they may have muscle mass loss and/or potential risk factors for malnutrition.

- Comprehensive assessment by an oncogeriatrician to help with optimal treatment choice should be conducted in patients identified as fragile by the oncologist using geriatric screening tools (G8 or VESI3).

- Patients (and caregivers) should be screened for psychological distress (anxiety, depression) and should be offered a referral for specialized support.

- Patients should be evaluated by a dentist, speech therapist, and physical therapist before, during, and after treatment.

- Survivors should be followed-up by the multidisciplinary team for identification of late toxicities, the potential need for further rehabilitation, and to get support for social relocation after treatment.

insurance coverage in the absence of public healthcare. ${ }^{8}$ Socially vulnerable and institutionalized patients present greater difficulties in treatment adherence. ${ }^{8}$ Even in developed countries, only about $50 \%$ of patients are able to return to work after 2 years and this is directly related to quality of life. ${ }^{62}$

The healthcare systems of developing countries predominantly have marked heterogeneity and difficulties in patient access, and as such, a high proportion of patients are diagnosed in advanced stages of SCCHN. Protocols for organ preservation usually apply to patients with a T2-3 N1 staging, while patients from developing countries are usually diagnosed at T4 N1-2 stage. ${ }^{61}$ In most of the available studies, $<30 \%$ of patients have advanced stage SCCHN. In this population, other factors associated with treatment adherence must be taken into account, such as the distance they have to travel to receive medical care, the availability of radiotherapy centers and chemotherapy administration units, and the experience of the surgical group. ${ }^{61}$

According to a Canadian study, the incidence of SCCHN was found to be higher in lower-income patient populations. However, the gap in incidence between the highest and the lowest incomes was narrowed for oropharyngeal cancer. ${ }^{63}$ Another study showed that the highest incidence of advanced stages occurred in low-income groups and in men living alone. The authors concluded that focus on the high risk of advanced stage SCCHN among vulnerable patients may be beneficial during referral and early diagnosis in order to improve outcomes in these patients. ${ }^{64}$

Socioeconomic factors not only have an impact during treatment, but also after completion of therapy. It is important to evaluate the patient's ability to return to work, identifying those patients who may require more intensive rehabilitation. Up to one-third of SCCHN patients in 
Brazil who are free of disease after 2 years of treatment cannot return to work. Advanced clinical stage, alcohol consumption, and low education level are independently associated with work disability. ${ }^{65}$ Moreover, education status, perceived economic status, and social security status have a significant effect on the quality of life of SCCHN patients after radiotherapy. ${ }^{66}$

\section{Multidisciplinary Support: Advance Nursing, Nutrition, Geriatric Assessment}

The care of patients with SCCHN is complex and requires the involvement of a wide range of services and experienced specialists for optimal treatment and follow-up, including specialized nursing care and clinical nutrition. ${ }^{47}$ Although oncogeriatrics does not appear in many of the guidelines, we expect that these specialists will gradually be incorporated into the multidisciplinary team. Other specialists and services involved in the management of patients with SCCHN are psychiatry, dentistry, speech and swallowing therapy, physical medicine and rehabilitation, and clinical social work. ${ }^{47}$

\section{Advanced Nursing}

Nursing staff are the backbone of managing patients with SCCHN, especially those receiving multimodal treatments, for which the participation of many specialists is necessary. In most cases, the success of treatment and coordination depends on nurses. Nurses are not only in charge of attending to patients, coordinating their treatment, and acting as a liaison between the patient and the multidisciplinary team, but also performs operational functions, referring patients, performing psychosocial evaluations, and providing health education once the multidisciplinary team has decided on a therapeutic strategy. ${ }^{67}$ At the level of care, nursing staff also offer emotional support, collaborate with other health professionals, and attend to the post-surgical needs and treatment-related toxicities. $^{67}$

\section{Nutrition}

The nutritional approach is fundamental in any oncologic treatment, but especially in patients with SCCHN. Several studies have demonstrated that poor nutritional status and severe weight loss during chemoradiotherapy are associated with increased morbidity and mortality. ${ }^{24,68-70}$ The difficulty in providing and maintaining adequate nutrition before and during treatment may result in the patient receiving suboptimal treatment or waiting too long before starting it, with the risk of becoming "unfit". ${ }^{24,61}$ However, despite intensive nutritional support from diagnosis to treatment for patients with advanced SCCHN, nutritional status deteriorates during multimodal treatment. Induction chemotherapy can improve nutritional status by reducing symptoms caused by the tumor itself that may limit oral intake. This improvement in nutritional status may help to minimize further deterioration during ongoing treatment. ${ }^{27}$ Nutritional assessment should be done not only at the time of diagnosis before receiving any treatment, but also daily after surgery, and later after chemoradiotherapy. ${ }^{67}$ In addition, nutritional assessment is advisable in patients who do not appear to be malnourished or have weight loss at diagnosis, as they may have loss of muscle mass and/or potential risk factors for malnutrition, either because of clinical dysphagia caused by the disease or because the volume and/ or location of the radiotherapy may pose a special risk of weight loss and malnutrition during treatment, especially when combined with chemotherapy). ${ }^{71,72}$

\section{Oncogeriatry}

A comprehensive geriatric assessment should be performed in all patients for whom treatment optimization is required due to their functional baseline status. This assessment includes the patient's quality of life, psychosocial support, independence, physical, and nutritional status, and cognitive and performance status. ${ }^{13}$ Through the comprehensive geriatric evaluation, the cost-benefit of whether or not to propose a treatment strategy is evaluated, and therefore, treatment can be adapted to patient's needs.

\section{Psychiatry}

Psychological distress is very common in SCCHN patients, with about $40 \%$ of patients experiencing depressive symptoms. Besides psychological distress, smoking and hazardous alcohol use are also common; even potential sexually transmitted diseases, like human papillomavirus-related SCCHN, are common. ${ }^{73-75}$ The suicide risk among SCCHN survivors is the higher than among other cancer survivors, and thus suicide contributes as a competing cause of non-cancerrelated death in survivors of SCCHN. ${ }^{76,77}$ Although the prevalence of emotional distress is high in patients with SCCHN, the referral rate for specialized support is very low. ${ }^{78}$ This led to the development of strategies for screening and referral of these patients, such as the anxiety and depressive symptoms questionnaires for adults with cancer developed by the American Society of Clinical Oncology (ASCO). ${ }^{79}$ In a prospective controlled trial, patients with $\mathrm{SCCHN}$ were randomized to a stepped-care or a standard-of-care approach. $^{80,81}$ The stepped-care protocol consisted of 
watchful waiting, guided self-help, problem-solving therapy, and psychotherapy and/or psychotropic medication. This study showed that the stepped-care approach not only led to a better patient quality of life, but was also a cost-effective strategy. ${ }^{80,81}$

\section{Dentistry}

Dentistry plays a very important role before (ie repair or removing compromised teeth), during (ie treatment of xerostomia and mucositis), and after treatment (ie looking for long-term toxicities during the follow-up phase). ${ }^{67,82}$ In fact, the NCCN guidelines recommend that all patients with SCCHN be evaluated by a dentist before, during, and after treatment. ${ }^{47}$ The establishment of a multidisciplinary team can improve patient access to dentistry services, and improved dental assessment leads to improvements in other endpoints. $^{83}$

\section{Speech and Swallow Rehabilitation}

Several guidelines recommend speech and swallow rehabilitation. All SCCHN patients should undergo pretreatment assessment of speech and swallowing, a program of prophylactic exercises, and continuous follow-up by a speech/swallow therapist after treatment, especially after laryngectomy. ${ }^{67}$ Developing a speech and swallow rehabilitation program improves patient satisfaction using a multidisciplinary approach. ${ }^{84}$

All these specialties are deeply interconnected. For example, the alleviation of dry mouth by a dentist improves swallowing function and nutritional status. ${ }^{85}$ Moreover, when patients are treated for depressive symptoms, their quality of life and swallowing function are improved. ${ }^{86}$ Studies have shown that a multidisciplinary approach lead to an improvement in OS for these patients. ${ }^{87,88}$ Another important part of the rehabilitation process is the patient's return to society and/or work.

\section{Conclusions}

Patients with SCCHN are often affected by comorbidities that hamper appropriate management and must be evaluated on an individual basis. Although cisplatin is an essential component of treatment in these patients, due to its acute and late toxicity, the administration of this drug is not suitable for all of them.

There are predictive patient factors that may help to determine whether cisplatin should be administered or not, or whether it should be administered with careful monitoring. These factors include biological age (rather than chronological age), performance status, weight loss, comorbidities, chronic viral infections, pre-chemotherapy toxicity, and even COVID19. The suitability of cisplatin must be discussed individually considering these predictive factors, even the combination of several of them.

The final decision must be based on a consensus by a multidisciplinary team in order to ensure that appropriate treatment and supportive measures are provided to these patients.

\section{Compliance with Ethics Guidelines}

This article does not contain any studies with human participants or animals performed by any of the authors.

\section{Acknowledgments}

The authors would like to thank Fernando Sánchez Barbero, $\mathrm{PhD}$, for providing medical writing assistance on behalf of Springer Healthcare, and Sarah Greig, $\mathrm{PhD}$, of Springer Healthcare Communications for editing the English language prior to submission. This medical writing assistance was funded by Merck KgaA, Darmstadt, Germany.

\section{Author contributions}

All authors have been involved in each phase of this project, which required their attendance to 5 online meetings. Under the guidance of Drs. Mesía and Falco, authors were asked to review current evidence of an assigned topic and present it to other authors during a series of online meetings. At each meeting, topics were presented followed by a discussion of the main gap areas on clinical practice and agreements of expert recommendations were reached. The topics discussed were: (1) factors to consider before establishing treatment (biological age, performance status, weight loss, comorbidities and toxicities), presented by Drs. Cacicedo, Soria, and Sowley; (2) contraindications for platinum-based chemotherapy due to toxicity to previous chemotherapy (Dr. Ticona), to COVID-19 (Dr. Aguilar) or chronic viral infections (Dr. Iglesias), to comorbidities and age (Drs. Galindo and RuedaDomínguez, respectively); (3) alternative treatments to high dose cisplatin (Dr. Pereira); and (4) other treatment related factors such as socio-economic factors (Dr. Ospina), and multidisciplinary approaches and team support (Drs. Taberna and de Oliveira).

All authors made substantial contributions to conception and design, acquisition of data, or analysis and interpretation of data; took part in drafting the article or revising it critically for important intellectual content; agreed to 
submit to the current journal; gave final approval of the version to be published; and agree to be accountable for all aspects of the work.

\section{Funding}

This work has been supported by Merck KgaA, Darmstadt, Germany.

\section{Disclosure}

None of the authors were compensated for this manuscript, but have received consultancy fees from the "TTCC-Grupo Español de Tratamiento de Tumores de Cabeza y Cuello" for the development of this consensus.

Ricard Mesía MD, PhD has received support for his advisory work from pharmaceutical companies including Merck, MSD, BMS, AstraZeneca, and Roche. He has also received fees for Speakers' bureau from Merck, MSD, Roche, and BMS.

Agustín Falco MD has received support for his advisory work from Merck, MSD, Biotoscana, Bayer and BMS. He has also received Speakers' bureau fees from Merck, MSD, BMS, Varifarma, and Raffo.

Thiago Bueno de Oliveira MD, PhD has received support for his advisory work from pharmaceutical companies including Merck Serono, Merck Sharp Dohme, MSD, BMS, Janssen, AstraZeneca, and Bayer. He has also received fees for Speakers' bureau from Merck, MSD, and BMS.

Aylen Vanessa Ospina MD has received support for advisory work from pharmaceutical companies including Merck, MSD, BMS and Roche. She has also received fees for Speakers' bureau from MSD, Roche and BMS.

Miguel Ángel Ticona MD has received support for his advisory work from Merck, MSD, Roche, Tecnofarma, Bayer, and Biotoscana. He has also received fees for Speaker's bureau from Merck, MSD, Tecnofarma, Bayer, and Takeda.

Marcos David Pereira MD has received support for his advisory work from Merck, MSD, and Novartis, and Speakers' bureau fees from Merck and MSD.

Antonio Rueda-Domínguez MD has received lectures and/or advisory fees from Merck, MSD, BMS, Takeda, Gilead, Novartis, Kern Pharma, and Roche.

Tannia Soria MD has received consultancy honoraria from Merck Ca, Abbott, Pfizer, MSD, Medicamenta, Roche, and Boehringer-Ingelheim. She has also received clinical studies fees from Roche, Tesaro, MSD, and Lilly. She has also received fees for her advisory work from Roche, Merck Ca, Pfizer, Novartis, BoehringerIngelheim, AstraZeneca, and MSD.

Miren Taberna MD, PhD has received support for her advisory work from pharmaceutical companies including Merck, MSD, and Nanobiotics. She has also received fees for Speakers' bureau from Merck, MSD, and BMS.

Lara Iglesias MD has received advisory board fees from Merck, MSD, BMS, Lilly, Roche, Sanofi, and Bayer.

Taysser Sowley MD has received support for her advisory work from Roche, MSD, Pfizer, Novartis, AstraZeneca, and Bayer. She has also received Speakers' bureau fees from Roche, MSD, Novartis, AstraZeneca, and Merck.

The remaining authors declare no conflicts of interest.

\section{References}

1. Adelstein DJ, Li Y, Adams GL, et al. An intergroup phase III comparison of standard radiation therapy and two schedules of concurrent chemoradiotherapy in patients with unresectable squamous cell head and neck cancer. J Clin Oncol. 2003;21(1):92-98. doi:10.1200/JCO.2003.01.008

2. Forastiere AA, Zhang Q, Weber RS, et al. Long-term results of RTOG 91-11: a comparison of three nonsurgical treatment strategies to preserve the larynx in patients with locally advanced larynx cancer. J Clin Oncol. 2013;31(7):845-852. doi:10.1200/JCO.2012.43.6097

3. Gillison ML, Trotti AM, Harris J, et al. Radiotherapy plus cetuximab or cisplatin in human papillomavirus-positive oropharyngeal cancer (NRG Oncology RTOG 1016): a randomised, multicentre, non-inferiority trial. Lancet. 2019;393(10166):40-50. doi:10.1016/ S0140-6736(18)32779-X

4. Vermorken JB, Mesía R, Rivera F, et al. Platinum-based chemotherapy plus cetuximab in head and neck cancer. $N$ Engl J Med. 2008;359 (11):1116-1127. doi:10.1056/NEJMoa0802656

5. Burtness B, Harrington KJ, Greil R, et al. Pembrolizumab alone or with chemotherapy versus cetuximab with chemotherapy for recurrent or metastatic squamous cell carcinoma of the head and neck (KEYNOTE-048): a randomised, open-label, Phase 3 study. Lancet. 2019;394(10212):1915-1928. doi:10.1016/S0140-6736(19)32591-7

6. Bernier J, Domenge C, Ozsahin M, et al. Postoperative irradiation with or without concomitant chemotherapy for locally advanced head and neck cancer. $N$ Engl J Med. 2004;350(19):1945-1952. doi:10.1056/NEJMoa032641

7. Ahn MJ, D'Cruz A, Vermorken JB, et al. Clinical recommendations for defining platinum unsuitable head and neck cancer patient populations on chemoradiotherapy: a literature review. Oral Oncol. 2016;53:10-16. doi:10.1016/j.oraloncology.2015.11.019

8. de Castro G Jr, Alves GV, Castro AF, et al. Criteria for eligibility to cisplatin in the curative treatment of head and neck cancer: consensus opinion from a panel of experts. Crit Rev Oncol Hematol. 2018;131:30-34. doi:10.1016/j.critrevonc.2018.08.009

9. Porceddu SV, Scotte F, Aapro M, et al. Treating patients with locally advanced squamous cell carcinoma of the head and neck unsuitable to receive cisplatin-based therapy. Front Oncol. 2020;9:1522. doi:10.3389/fonc. 2019.01522

10. Syrigos KN, Karachalios D, Karapanagiotou EM, Nutting CM, Manolopoulos L, Harrington KJ. Head and neck cancer in the elderly: an overview on the treatment modalities. Cancer Treat Rev. 2009;35 (3):237-245. doi:10.1016/j.ctrv.2008.11.002 
11. Porceddu SV, Haddad RI. Management of elderly patients with locoregionally confined head and neck cancer. Lancet Oncol. 2017;18(5):e274-e283. doi:10.1016/S1470-2045(17)30229-2

12. Szturz P, Bossi P, Vermorken JB. Systemic treatment in elderly head and neck cancer patients: recommendations for clinical practice. $\mathrm{Curr}$ Opin Otolaryngol Head Neck Surg. 2019;27(2):142-150. doi:10.1097/MOO.0000000000000526

13. Maggiore R, Zumsteg ZS, BrintzenhofeSzoc K, et al. The older adult with locoregionally advanced head and neck squamous cell carcinoma: knowledge gaps and future direction in assessment and treatment. Int $J$ Radiat Oncol Biol Phys. 2017;98(4):868-883. doi:10.1016/j.ijrobp.2017.02.022

14. Antonio M, Honorato M, Plana M, et al. 934P comparing frailty screening tools for older head and neck cancer patients. Ann Oncol. 2020;31(Suppl 4):S670. doi:10.1016/j. annonc.2020.08.1049

15. Lacas B, Carmel A, Landais C, et al. Meta-analysis of chemotherapy in head and neck cancer (MACH-NC): an update on 107 randomized trials and 19805 patients, on behalf of MACH-NC group. Radiother Oncol. 2021;156:281-293. doi:10.1016/j. radonc.2021.01.013

16. Guigay J, Auperin A, Mertens C, et al. Personalized treatment according to geriatric assessment in 1st line recurrent and/or metastatic (R/M) Head and Neck Squamous Cell Cancer (HNSCC) patients aged 70 or over. ELAN (ELderly heAd and Neck cancer) FIT and UNFIT trials. Ann Oncol. 2019;30(Suppl5):Abstract3250. doi:10.1093/annonc/mdz252.002

17. Argiris A, Li Y, Murphy BA, Langer CJ, Forastiere AA. Outcome of elderly patients with recurrent or metastatic head and neck cancer treated with cisplatin-based chemotherapy. J Clin Oncol. 2004;22 (2):262-268. doi:10.1200/JCO.2004.08.039

18. Ward MC, Reddy CA, Adelstein DJ, Koyfman SA. Use of systemic therapy with definitive radiotherapy for elderly patients with head and neck cancer: a national cancer data base analysis. Cancer. 2016;122(22):3472-3483. doi:10.1002/cncr.30214

19. Woody NM, Ward MC, Koyfman SA, et al. Adjuvant chemoradiation after surgical resection in elderly patients with high-risk squamous cell carcinoma of the head and neck: a national cancer database analysis. Int J Radiat Oncol Biol Phys. 2017;98(4):784-792. doi:10.1016/j.ijrobp.2017.03.019

20. Cooper JS, Pajak TF, Forastiere AA, et al. Postoperative concurrent radiotherapy and chemotherapy for high-risk squamous-cell carcinoma of the head and neck. $N$ Engl $J$ Med. 2004;350 (19):1937-1944. doi:10.1056/NEJMoa032646

21. Saraswathula A, Chen MM, Colevas AD, Divi V. Assessing care value for older patients receiving radiotherapy with or without cisplatin or cetuximab for locoregionally advanced head and neck cancer. JAMA Otolaryngol Head Neck Surg. 2019;145 (12):1160-1167. doi:10.1001/jamaoto.2019.2381

22. Muller von der Grun J, Martin D, Stover T, Ghanaati S, Rodel C, Balermpas P. Chemoradiotherapy as definitive treatment for elderly patients with head and neck cancer. Biomed Res Int. 2018;2018:3508795. doi:10.1155/2018/3508795

23. Machtay M, Moughan J, Trotti A, et al. Factors associated with severe late toxicity after concurrent chemoradiation for locally advanced head and neck cancer: an RTOG analysis. $J$ Clin Oncol. 2008;26(21):3582-3589. doi:10.1200/JCO.2007.14.8841

24. Capuano G, Grosso A, Gentile PC, et al. Influence of weight loss on outcomes in patients with head and neck cancer undergoing concomitant chemoradiotherapy. Head Neck. 2008;30(4):503-508. doi:10.1002/hed.20737
25. Pawar D, Ahmed R, Chaudhari S. A survey of on platinum ineligible in head and neck cancer patients in India. Ann Oncol. 2017;28(Suppl 10):Abstract520P. doi:10.1093/annonc/mdx676.019

26. Ghadjar P, Hayoz S, Zimmermann F, et al. Impact of weight loss on survival after chemoradiation for locally advanced head and neck cancer: secondary results of a randomized phase III trial (SAKK 10/ 94). Radiat Oncol. 2015;10(1):21. doi:10.1186/s13014-014-0319-y

27. Arribas L, Hurtós L, Taberna M, et al. Nutritional changes in patients with locally advanced head and neck cancer during treatment. Oral Oncol. 2017;71:67-74. doi:10.1016/j.oraloncology.2017.06.003

28. Talwar B, Donnelly R, Skelly R, Donaldson M. Nutritional management in head and neck cancer: United Kingdom national multidisciplinary guidelines. $J$ Laryngol Otol. 2016;130(S2):S32-S40. doi:10.1017/S0022215116000402

29. Langer T, Am Zehnhoff-Dinnesen A, Radtke S, Meitert J, Zolk O. Understanding platinum-induced ototoxicity. Trends Pharmacol Sci. 2013;34(8):458-469. doi:10.1016/j.tips.2013.05.006

30. McWhinney SR, Goldberg RM, McLeod HL. Platinum neurotoxicity pharmacogenetics. Mol Cancer Ther. 2009;8(1):10-16. doi:10.1158/ 1535-7163.MCT-08-0840

31. Charlson ME, Pompei P, Ales KL, MacKenzie CR. A new method of classifying prognostic comorbidity in longitudinal studies: development and validation. J Chronic Dis. 1987;40(5):373-383. doi:10.1016/00219681(87)90171-8

32. Charlson ME, Charlson RE, Peterson JC, Marinopoulos SS, Briggs WM, Hollenberg JP. The Charlson comorbidity index is adapted to predict costs of chronic disease in primary care patients. $J$ Clin Epidemiol. 2008;61(12):1234-1240. doi:10.1016/j.jclinepi.2008.01.006

33. Shih CA, Chen WC, Yu HC, et al. Risk of severe acute exacerbation of chronic HBV infection cancer patients who underwent chemotherapy and did not receive anti-viral prophylaxis. PLoS One. 2015;10 (8):e0132426. doi:10.1371/journal.pone.0132426

34. Liu PP. Cardiorenal syndrome in heart failure: a cardiologist's perspective. Can J Cardiol. 2008;24(SupplB):25B-29B. doi:10.1016/ S0828-282X(08)71027-4

35. Szturz P, Cristina V, Herrera Gómez RG, Bourhis J, Simon C, Vermorken JB. Cisplatin eligibility issues and alternative regimens in locoregionally advanced head and neck cancer: recommendations for clinical practice. Front Oncol. 2019;9:464. doi:10.3389/ fonc. 2019.00464

36. Rudek MA, Flexner C, Ambinder RF. Use of antineoplastic agents in patients with cancer who have HIV/AIDS. Lancet Oncol. 2011;12 (9):905-912. doi:10.1016/S1470-2045(11)70056-0

37. Kawsar HI, Shahnewaz J, Gopalakrishna KV, Spiro TP, Daw HA. Hepatitis B reactivation in cancer patients: role of prechemotherapy screening and antiviral prophylaxis. Clin Adv Hematol Oncol. 2012;10(6):370-378.

38. Mahale P, Sturgis EM, Tweardy DJ, Ariza-Heredia EJ, Torres HA. Association between hepatitis $\mathrm{C}$ virus and head and neck cancers. $J$ Natl Cancer Inst. 2016;108(8):djw035. doi:10.1093/jnci/djw035

39. Rangel JB, Thuler LCS, Pinto J. Prevalence of hepatitis C virus infection and its impact on the prognosis of head and neck cancer patients. Oral Oncol. 2018;87:138-143. doi:10.1016/j.oraloncology.2018.10.024

40. Lee LY, Cazier JB, Angelis V, et al. COVID-19 mortality in patients with cancer on chemotherapy or other anticancer treatments: a prospective cohort study. Lancet. 2020;395(10241):1919-1926. doi:10.1016/S0140-6736(20)31173-9

41. de Felice F, Polimeni A, Tombolini V. The impact of coronavirus (COVID-19) on head and neck cancer patients' care. Radiother Oncol. 2020;147:84-85. doi:10.1016/j.radonc.2020.03.020 
42. Chaves ALF, Castro AF, Marta GN, et al. Emergency changes in international guidelines on treatment for head and neck cancer patients during the COVID-19 pandemic. Oral Oncol. 2020;107:104734. doi:10.1016/j.oraloncology.2020.104734

43. Grupo Español del Tratamiento de Tumores de Cabeza y Cuello. Recomendaciones de tratamiento del cáncer de cabeza y cuello (pandemia SARS-CoV-2); 2020.

44. Gietema JA, Meinardi MT, Messerschmidt J, et al. Circulating plasma platinum more than 10 years after cisplatin treatment for testicular cancer. Lancet. 2000;355(9209):1075-1076. doi:10.1016/ S0140-6736(00)02044-4

45. Strojan P, Vermorken JB, Beitler JJ, et al. Cumulative cisplatin dose in concurrent chemoradiotherapy for head and neck cancer: a systematic review. Head Neck. 2016;38(Suppl 1):E2151-2158. doi:10.1002/hed.24026

46. Makrilia N, Syrigou E, Kaklamanos I, Manolopoulos L, Saif MW. Hypersensitivity reactions associated with platinum antineoplastic agents: a systematic review. Met Based Drugs. 2010;2010:1-11. doi: $10.1155 / 2010 / 207084$

47. National Comprehensive Cancer Network. NCCN clinical practice guidelines in oncology. head and neck cancers. Version 1.2021; November 9, 2020. Available from: https://www.nccn.org/professionals/physician_gls/ pdf/head-and-neck.pdf. Accessed November 16, 2020.

48. Mesia R, Iglesias L, Lambea J, et al. SEOM clinical guidelines for the treatment of head and neck cancer (2020). Clin Transl Oncol. 2021;23(5):913-921.

49. Denis F, Garaud P, Bardet E, et al. Final results of the 94-01 French head and neck oncology and radiotherapy group randomized trial comparing radiotherapy alone with concomitant radiochemotherapy in advanced-stage oropharynx carcinoma. J Clin Oncol. 2004;22 (1):69-76. doi:10.1200/JCO.2004.08.021

50. Hanemaaijer SH, Kok IC, Fehrmann RSN, et al. Comparison of carboplatin with 5 -fluorouracil vs. cisplatin as concomitant chemoradiotherapy for locally advanced head and neck squamous cell carcinoma. Front Oncol. 2020;10:761. doi:10.3389/fonc.2020.00761

51. Guan J, Li Q, Zhang Y, et al. A meta-analysis comparing cisplatin-based to carboplatin-based chemotherapy in moderate to advanced squamous cell carcinoma of head and neck (SCCHN). Oncotarget. 2016;7(6):7110-7119. doi:10.18632/oncotarget.6858

52. Bonner JA, Harari PM, Giralt J, et al. Radiotherapy plus cetuximab for squamous-cell carcinoma of the head and neck. $N$ Engl $J$ Med. 2006;354(6):567-578. doi:10.1056/NEJMoa053422

53. Bonner JA, Harari PM, Giralt J, et al. Radiotherapy plus cetuximab for locoregionally advanced head and neck cancer: 5-year survival data from a phase 3 randomised trial, and relation between cetuximab-induced rash and survival. Lancet Oncol. 2010;11 (1):21-28. doi:10.1016/S1470-2045(09)70311-0

54. Lee YG, Kang EJ, Keam B, et al. Treatment strategy and outcomes in locally advanced head and neck squamous cell carcinoma: a nationwide retrospective cohort study (KCSG HN13-01). BMC Cancer. 2020;20(1):813. doi:10.1186/s12885-020-07297-z

55. Hitt R, Irigoyen A, Cortes-Funes H, et al. Phase II study of the combination of cetuximab and weekly paclitaxel in the first-line treatment of patients with recurrent and/or metastatic squamous cell carcinoma of head and neck. Ann Oncol. 2012;23(4):1016-1022. doi:10.1093/annonc/mdr367

56. Bachaud JM, Cohen-Jonathan E, Alzieu C, David JM, Serrano E, DalySchveitzer N. Combined postoperative radiotherapy and weekly cisplatin infusion for locally advanced head and neck carcinoma: final report of a randomized trial. Int J Radiat Oncol Biol Phys. 1996;36(5):999-1004. doi:10.1016/S0360-3016(96)00430-0

57. Noronha V, Joshi A, Patil VM, et al. Once-a-week versus once-every3-weeks cisplatin chemoradiation for locally advanced head and neck cancer: a phase III randomized noninferiority trial. J Clin Oncol. 2018;36(11):1064-1072. doi:10.1200/JCO.2017.74.9457
58. Kiyota N, Tahara M, Fujii H, et al. Phase II/III trial of post-operative chemoradiotherapy comparing 3-weekly cisplatin with weekly cisplatin in high-risk patients with squamous cell carcinoma of head and neck (JCOG1008). J Clin Oncol. 2020;38(15 Suppl):Abstract 6502. doi:10.1200/JCO.2020.38.15_suppl.6502

59. Tsan DL, Lin CY, Kang CJ, et al. The comparison between weekly and three-weekly cisplatin delivered concurrently with radiotherapy for patients with postoperative high-risk squamous cell carcinoma of the oral cavity. Radiat Oncol. 2012;7(1):215. doi:10.1186/1748-717X-7-215

60. Lacas B, Bourhis J, Overgaard J, et al. Role of radiotherapy fractionation in head and neck cancers (MARCH): an updated meta-analysis. Lancet Oncol. 2017;18(9):1221-1237. doi:10.1016/ S1470-2045(17)30458-8

61. Sanabria A, Domenge C, D'Cruz A, Kowalski LP. Organ preservation protocols in developing countries. Curr Opin Otolaryngol Head Neck Surg. 2010;18(2):83-88. doi:10.1097/MOO.0b013e3283378f40

62. Isaksson J, Wilms T, Laurell G, Fransson P, Ehrsson YT. Meaning of work and the process of returning after head and neck cancer. Support Care Cancer. 2016;24(1):205-213. doi:10.1007/s00520-015-2769-7

63. Hwang E, Johnson-Obaseki S, McDonald JT, Connell C, Corsten M. Incidence of head and neck cancer and socioeconomic status in Canada from 1992 to 2007. Oral Oncol. 2013;49(11):1072-1076. doi:10.1016/j.oraloncology.2013.08.002

64. Olsen MH, Boje CR, Kjaer TK, et al. Socioeconomic position and stage at diagnosis of head and neck cancer - a nationwide study from DAHANCA. Acta Oncol. 2015;54(5):759-766. doi:10.3109/ 0284186X.2014.998279

65. Vartanian JG, Carvalho AL, Toyota J, Kowalski IS, Kowalski LP. Socioeconomic effects of and risk factors for disability in long-term survivors of head and neck cancer. Arch Otolaryngol Head Neck Surg. 2006;132(1):32-35. doi:10.1001/archotol.132.1.32

66. Demiral AN, Sen M, Demiral Y, Kinay M. The effect of socioeconomic factors on quality of life after treatment in patients with head and neck cancer. Int J Radiat Oncol Biol Phys. 2008;70 (1):23-27. doi:10.1016/j.ijrobp.2007.05.056

67. Taberna M, Gil Moncayo F, Jané-Salas E, et al. The Multidisciplinary Team (MDT) approach and quality of care. Front Oncol. 2020;10:85. doi: $10.3389 /$ fonc. 2020.00085

68. Jager-Wittenaar H, Dijkstra PU, Vissink A, van der Laan BF, van Oort RP, Roodenburg JL. Critical weight loss in head and neck cancer-prevalence and risk factors at diagnosis: an explorative study. Support Care Cancer. 2007;15(9):1045-1050. doi:10.1007/ s00520-006-0212-9

69. Lees J. Incidence of weight loss in head and neck cancer patients on commencing radiotherapy treatment at a regional oncology centre. Eur J Cancer Care (Engl). 1999;8(3):133-136. doi:10.1046/j.13652354.1999.00156.x

70. Salas S, Deville JL, Giorgi R, et al. Nutritional factors as predictors of response to radio-chemotherapy and survival in unresectable squamous head and neck carcinoma. Radiother Oncol. 2008;87 (2):195-200. doi:10.1016/j.radonc.2008.02.011

71. Paccagnella A, Morello M, Da Mosto MC, et al. Early nutritional intervention improves treatment tolerance and outcomes in head and neck cancer patients undergoing concurrent chemoradiotherapy. Support Care Cancer. 2010;18(7):837-845. doi:10.1007/s00520-009-0717-0

72. Ferrao B, Neves PM, Santos T, Capelas ML, Makitie A, Ravasco P. Body composition changes in patients with head and neck cancer under active treatment: a scoping review. Support Care Cancer. 2020;28(10):4613-4625. doi:10.1007/s00520-020-05487-w

73. McCarter K, Baker AL, Britton B, et al. Smoking, drinking, and depression: comorbidity in head and neck cancer patients undergoing radiotherapy. Cancer Med. 2018;7(6):2382-2390. doi:10.1002/ cam4.1497

74. Sehlen S, Lenk M, Herschbach P, et al. Depressive symptoms during and after radiotherapy for head and neck cancer. Head Neck. 2003;25 (12):1004-1018. doi:10.1002/hed.10336 
75. Reich M, Licitra L, Vermorken JB, et al. Best practice guidelines in the psychosocial management of HPV-related head and neck cancer: recommendations from the European head and neck cancer society's make sense campaign. Ann Oncol. 2016;27(10):1848-1854. doi:10.1093/annonc/mdw272

76. Osazuwa-Peters N, Simpson MC, Zhao L, et al. Suicide risk among cancer survivors: head and neck versus other cancers. Cancer. 2018;124(20):4072-4079. doi:10.1002/cncr.31675

77. Yu GP, Mehta V, Branovan D, Huang Q, Schantz SP. Non-cancerrelated deaths from suicide, cardiovascular disease, and pneumonia in patients with oral cavity and oropharyngeal squamous carcinoma. Arch Otolaryngol Head Neck Surg. 2012;138(1):25-32. doi:10.1001/archoto.2011.236

78. Verdonck-de Leeuw IM, de Bree R, Keizer AL, et al. Computerized prospective screening for high levels of emotional distress in head and neck cancer patients and referral rate to psychosocial care. Oral Oncol. 2009;45(10):e129-e133. doi:10.1016/j.oraloncology.2009.01.012

79. Andersen BL, DeRubeis RJ, Berman BS, et al. Screening, assessment, and care of anxiety and depressive symptoms in adults with cancer: an American society of clinical oncology guideline adaptation. J Clin Oncol. 2014;32(15):1605-1619. doi:10.1200/ JCO.2013.52.4611

80. Krebber AM, Leemans CR, de Bree R, et al. Stepped care targeting psychological distress in head and neck and lung cancer patients: a randomized clinical trial. BMC Cancer. 2012;12(1):173. doi:10.1186/1471-2407-12-173

81. Krebber AM, Jansen F, Witte BI, et al. Stepped care targeting psychological distress in head and neck cancer and lung cancer patients: a randomized, controlled trial. Ann Oncol. 2016;27(9):1754-1760. doi:10.1093/annonc/mdw230
82. Villa A, Akintoye SO. Dental management of patients who have undergone oral cancer therapy. Dent Clin North Am. 2018;62 (1):131-142. doi:10.1016/j.cden.2017.08.010

83. Kelly SL, Jackson JE, Hickey BE, Szallasi FG, Bond CA. Multidisciplinary clinic care improves adherence to best practice in head and neck cancer. Am J Otolaryngol. 2013;34(1):57-60. doi:10.1016/j.amjoto.2012.08.010

84. Starmer HM, Ayoub N, Byward C, et al. The impact of developing a speech and swallow rehab program: improving patient satisfaction and multidisciplinary care. Laryngoscope. 2017;127(11):2578-2581.

85. Nuchit S, Lam-Ubol A, Paemuang W, et al. Alleviation of dry mouth by saliva substitutes improved swallowing ability and clinical nutritional status of post-radiotherapy head and neck cancer patients: a randomized controlled trial. Support Care Cancer. 2020;28 (6):2817-2828. doi:10.1007/s00520-019-05132-1

86. Lin BM, Starmer HM, Gourin CG. The relationship between depressive symptoms, quality of life, and swallowing function in head and neck cancer patients 1 year after definitive therapy. Laryngoscope. 2012;122(7):1518-1525. doi:10.1002/lary.23312

87. Liao CT, Kang CJ, Lee LY, et al. Association between multidisciplinary team care approach and survival rates in patients with oral cavity squamous cell carcinoma. Head Neck. 2016;38(Suppl 1): E1544-E1553. doi:10.1002/hed.24276

88. Light T, Rassi EE, Maggiore RJ, et al. Improving outcomes in veterans with oropharyngeal squamous cell carcinoma through implementation of a multidisciplinary clinic. Head Neck. 2017;39 (6):1106-1112. doi:10.1002/hed.24721

\section{Publish your work in this journal}

Cancer Management and Research is an international, peer-reviewed open access journal focusing on cancer research and the optimal use of preventative and integrated treatment interventions to achieve improved outcomes, enhanced survival and quality of life for the cancer patient.
The manuscript management system is completely online and includes a very quick and fair peer-review system, which is all easy to use. Visit http://www.dovepress.com/testimonials.php to read real quotes from published authors. 